Article

\title{
Correcting the Bias in the Practitioner Black-Scholes Method
}

\author{
Yun Yin ${ }^{1}$ and Peter G. Moffatt ${ }^{2, * \mathbb{D}}$ \\ 1 School of Business, Macau University of Science and Technology, Avenida Wai Long, Taipa, Macau; \\ yyin@must.edu.mo \\ 2 School of Economics, University of East Anglia, Norwich Research Park, Norwich, Norfolk NR4 7TJ, UK \\ * Correspondence: p.moffatt@uea.ac.uk
}

Received: 16 August 2019; Accepted: 23 September 2019; Published: 26 September 2019

\begin{abstract}
We address a number of technical problems with the popular Practitioner Black-Scholes (PBS) method for valuing options. The method amounts to a two-stage procedure in which fitted values of implied volatilities (IV) from a linear regression are plugged into the Black-Scholes formula to obtain predicted option prices. Firstly we ensure that the prediction from stage one is positive by using log-linear regression. Secondly, we correct the bias that results from the transformation applied to the fitted values (i.e., the Black-Scholes formula) being a highly non-linear function of implied volatility. We apply the smearing technique in order to correct this bias. An alternative means of implementing the PBS approach is to use the market option price as the dependent variable and estimate the parameters of the IV equation by the method of non-linear least squares (NLLS). A problem we identify with this method is one of model incoherency: the IV equation that is estimated does not correspond to the set of option prices used to estimate it. We use the Monte Carlo method to verify that (1) standard PBS gives biased option values, both in-sample and out-of-sample; (2) using standard (log-linear) PBS with smearing almost completely eliminates the bias; (3) NLLS gives biased option values, but the bias is less severe than with standard PBS. We are led to conclude that, of the range of possible approaches to implementing PBS, log-linear PBS with smearing is preferred on the basis that it is the only approach that results in valuations with negligible bias.
\end{abstract}

Keywords: option pricing; Practitioner Black-Scholes method; smearing; non-linear least squares; Monte Carlo

JEL Classification: C58; G12

\section{Introduction}

The Practitioner Black-Scholes (PBS) method (Dumas et al. 1998; Christoffersen and Jacobs 2004) has become a very popular as a benchmark option pricing method, against which other pricing methods can usefully be compared. ${ }^{1}$ The method amounts to the use of a cross-section sample of market option prices to estimate the implied volatility surface, that is, to estimate the parameters of an equation showing implied volatility (IV) as a function of strike price and time to expiry. The estimated IV equation can then be used to predict option prices, either within-sample or out-of-sample. The purpose

1 Examples of option pricing models for which a comparison against the benchmark of PBS is likely to be very useful are: Bakshi et al. (1997) SVSI-J model which allows volatility, interest rates, and jumps all to be stochastic; Heston and Nandi (2000) model built on the assumption of a GARCH process in the underlying index; and Duan et al. (2006) models built on even more general assumptions such as GJR-GARCH (TGARCH) and EGARCH. 
of this paper is to raise a number of technical problems arising with the PBS method, and to propose methods for overcoming them.

There are a number of possible ways of implementing the PBS method. One, which we shall call "standard PBS", is a two-stage approach: (1) use the cross-section of options to estimate a linear regression model with IV as the dependent variable, and strike price, time to expiry, and their squares and cross-product, as explanatory variables; (2) plug the fitted values from this regression into the Black-Scholes formula to obtain predicted option prices.

One immediate problem is that while IV is always positive, there is no guarantee that the prediction of IV from the stage-one regression will be positive. We address this problem by using a log-linear regression in stage one. A possibly more serious problem relates to stage two: as pointed out by Christoffersen and Jacobs (2004), the two-stage method yields biased predictions of option valuations, for the simple reason that the transformation being applied to the fitted values (i.e., the Black-Scholes formula) is a non-linear function of implied volatility. In this paper, we apply the smearing technique (Duan 1983) in order to correct this bias. The smearing technique makes use of the residuals from the IV regression as well as the fitted values and is classified as a non-parametric technique since it is valid whatever the actual distribution of the error term in the IV equation.

An alternative means of implementing the PBS approach is to use the market option price as the dependent variable and estimate the parameters of the IV equation by the method of non-linear least squares (NLLS). A problem we identify with this method is one of model incoherency. The model that underlies the method of NLLS can be shown to be incoherent in the sense that the IV equation that is estimated does not correspond to the set of option prices used to estimate it. Hence NLLS estimation of the IV equation is inconsistent. This may be verified by applying the Hausman test to establish that the IV equation estimated using NLLS is systematically different from that estimated using OLS with IV data.

In this paper, the Monte Carlo method is used to verify that: standard PBS gives biased option values; using standard (log-linear) PBS with smearing corrects the bias, both in-sample and out-of-sample; NLLS gives biased option values, but the bias is less severe than with standard PBS. We also compare other standard measures of performance (MAE and MSE) for each of these prediction measures. We are led to conclude that, of the range of possible approaches to implementing PBS, standard (log-linear) PBS with smearing is preferred on the basis that it is the only approach that results in unbiased valuations, and also performs well in terms of precision.

In Section 2, we outline the PBS method. In Section 3, we outline Duan (1983) smearing method, and explain how it can be applied to the PBS method. In Section 4, we consider how the IV equation can be estimated by NLLS applied to option prices. In Section 5 we consider the problem of estimating the IV equation using data from a cross-section of options traded on a particular day. In Section 6 we use estimates from one of the IV equations estimated in Section 5 to design a data generating process (DGP) for a Monte Carlo experiment whose purpose is to evaluate the relative performance of the various prediction methods considered earlier. The principal focus is on which method yields unbiased predictions. Section 7 concludes.

\section{The PBS Method}

There are three steps in the implementation of the PBS model. The first step is to compute the implied volatility of each option in the sample using (the inverse of) the Black-Scholes formula. That is, to find the implied volatility of call option $i$, we compute the value of $\sigma_{i}$ that causes the following equality to be satisfied:

$$
C_{i}=C_{i}^{B S}\left(\sigma_{i}\right)
$$


where $C_{i}$ is the market price of call option $i$, and $C_{i}^{B S}(\cdot)$ is the Black-Scholes valuation of call option $i^{2}$

Second, simple ordinary least squares (OLS) is used to run a regression of implied volatilities on different polynomials in time-to-expiry and strike price. This regression model is sometimes referred to as the "DFW implied volatility (IV) equation", having been introduced by Dumas et al. (1998). The most general IV equation appearing in the literature is

$$
\sigma(\theta)=\theta_{0}+\theta_{1} K+\theta_{2} K^{2}+\theta_{3} \tau+\theta_{4} \tau^{2}+\theta_{5} K \tau+\varepsilon_{I V}
$$

where $\sigma$ is implied volatility, $K$ is the strike price, and $\tau$ is time to expiry.

Finally, the fitted values for IV are obtained as

$$
\sigma(\hat{\theta})=\hat{\theta}_{0}+\hat{\theta}_{1} K+\hat{\theta}_{2} K^{2}+\hat{\theta}_{3} \tau+\hat{\theta}_{4} \tau^{2}+\hat{\theta}_{5} K \tau
$$

where hats indicate OLS estimates. These fitted values are plugged back into the Black-Scholes formula to obtain the PBS prices.

$$
C^{P B S}=C^{B S}(\sigma(\hat{\theta}))
$$

There is a logical problem with (2), since it does not restrict the implied volatility to be positive. Hence there is a possibility that the fitted values (3) take negative values and this would result in meaningless predictions of option prices when (4) is applied. We will address this problem by assuming a log-linear form for the IV equation ${ }^{3}$ :

$$
\ln [\sigma(\theta)]=\theta_{0}+\theta_{1} K+\theta_{2} K^{2}+\theta_{3} \tau+\theta_{4} \tau^{2}+\theta_{5} K \tau+\varepsilon_{I V}
$$

Let the vector of estimates from (5) be $\hat{\theta}_{\log I V}$. Given fitted values from (5), $\ln \left[\sigma\left(\hat{\theta}_{\log I V}\right)\right]$, an obvious procedure for obtaining predicted option prices is to use the following analogue of (4);

$$
C^{P B S, \log I V}=C^{B S}\left[\exp \left(\ln \left[\sigma\left(\hat{\theta}_{\log I V}\right)\right]\right)\right]
$$

Christoffersen and Jacobs (2004, p. 298), make a very important point when outlining the PBS procedure: "It is clear that simply plugging $\sigma\left(\theta_{I V}\right)$ into the Black-scholes formula will yield a biased estimate of the observed call price. While OLS will ensure that $E\left[\varepsilon_{I V}\right]=0$, the non-linearity of the dollar option price in volatility and thus in $\varepsilon_{I V}$ implies that $E[C] \neq C^{B S}\left(\sigma\left(\theta_{I V}\right)\right)$." This problem arises if either (4) or (6) is used, and is because (4) and (6) are both non-linear functions of the predicted implied volatility.

To our knowledge, no correction has ever been made for this problem. This motivates us to apply the smearing technique (Duan 1983) to correct the bias. This approach to correcting the bias was first suggested by Yin (2018).

\section{The Smearing Method in Prediction}

Consider the regression model:

$$
y_{i}=x_{i}^{\prime} \beta+u_{i} \quad i=1, \ldots, n
$$

2 The Black-Scholes formula (Hull 2011) also contains as arguments: current price of the underlying; strike price; time-to-expiry, risk-free rate. Since the focus here is on volatility, we suppress these arguments and express the option value as a function of only the volatility of the underlying, $\sigma$.

3 The log-linear form (5) is clearly a new and untested class of implied volatility function. However, since $\ln (\cdot)$ is a monotonically increasing function, any non-monotonic pattern in the original IV function (such as the well-known "volatility smile") is obviously reproduced (albeit with a different shape) when the log of IV is used. Other authors (e.g., Andreou et al. 2014) address the negativity problem by applying a lower bound on predicted IV, using for example $\max (\sigma(\hat{\theta}), 0.01)$ in place of $\sigma(\hat{\theta})$ in (4). On Monte Carlo evidence, we find that this approach tends to exacerbate biases in option values. For this reason, and also because the lower-bound correction is ad-hoc, we prefer the log-linear approach. 
Suppose that Equation (7) is estimated by OLS, resulting in predictions $\hat{y}_{i}$ and residuals $\hat{u}_{i}$. But assume that we are interested in predicting not $y$, but some non-linear function $f(y)$ of $y$. We could just use $f(\hat{y})$ for this. However, this gives biased predictions unless the function $f(\cdot)$ is linear.

Duan (1983) proposed the "smearing" formula which is as follows:

$$
\hat{f_{i}}=\frac{1}{n} \sum_{j=1}^{n} f\left(\hat{y}_{i}+\hat{u}_{j}\right)
$$

The important feature of the smearing formula (8) is that each observation (i) is predicted using the mean of a quantity involving the OLS prediction of $y_{i}$ and all of the $n$ residuals $\left(\hat{u}_{j}, j=1, \ldots, n\right)$. Consistency of the smearing estimator (8) is proved by Duan (1983). It is classified as a non-parametric method, since it gives rise to consistent predictions whatever the distribution of the error term $(u)$ in the regression model.

To see exactly how smearing may be applied to the PBS model, consider regression Equation (2) above. The fitted values from this regression, $\sigma_{i}(\hat{\theta})$, are given by (3). Further, let the residuals be $\hat{\varepsilon}_{i}$. The prediction of the price of option $i$, with smearing, is given by

$$
C_{i}^{P B S, S M}=\frac{1}{n} \sum_{j=1}^{n} C_{i}^{B S}\left(\sigma_{i}(\hat{\theta})+\hat{\varepsilon}_{j}\right)
$$

As noted in Section 2, a problem with (3) is that it is possible for the predicted IV to be negative, giving rise to meaningless predictions. With (9), this problem is more serious. Even in a situation in which all of the predicted IV's are positive, it is highly likely that the lowest predicted IV's become negative when negative residuals are added to them, making some terms of the sum in (9) meaningless.

This problem is avoided if smearing is instead applied to (5) and (6). Letting the residuals from (5) be $\hat{\varepsilon}_{i}$, the prediction with smearing is

$$
C_{i}^{P B S, \log I V, S M}=\frac{1}{n} \sum_{j=1}^{n} C_{i}^{B S}\left[\exp \left(\ln \left[\sigma_{i}\left(\hat{\theta}_{\log I V}\right)\right]+\hat{\varepsilon}_{j}\right)\right]
$$

In (10) all terms contained in the outermost brackets are positive, as required. In Appendix B, we provide the STATA code that is required to implement (10) using a cross-section sample of call options.

The smearing technique can also be applied to the problem of out-of-sample prediction. Consider option $n+1$ that does not appear in the estimation sample. Equation (10) can be extended to predict the value of option $n+1$, as follows:

$$
C_{n+1}^{P B S, \log I V, S M}=\frac{1}{n+1} \sum_{j=1}^{n+1} C_{n+1}^{B S}\left[\exp \left(\ln \left[\sigma_{n+1}\left(\hat{\theta}_{\log I V}\right)\right]+\hat{\varepsilon}_{j}\right)\right]
$$

where $\hat{\varepsilon}_{n+1}=0$, that is, the residual for the out-of-sample option is set to zero in the smearing formula.

\section{Estimation of the IV Equation by NLLS on Option Price Data}

An alternative method for estimating the parameters of the IV equation, (2) or (5), is to apply non-linear least squares to the option price data. Again consider a cross section of call option prices $C_{i}, i=1, \ldots, n$. The NLLS estimator of the IV equation parameters, $\theta$, defined in (2) above, is given by

$$
\hat{\theta}_{N L L S}=\underset{\theta}{\operatorname{argmin}} \sum_{i=1}^{n}\left[C_{i}-C_{i}^{B S}\left(\sigma_{i}(\theta)\right)\right]^{2}
$$

A problem we identify with this method is one of model incoherency. The model that underlies the method of NLLS can be shown to be incoherent in the sense that the IV equation that is estimated does not correspond to the set of option prices used to estimate it. Hence NLLS estimation of the IV equation is inconsistent. 
The problem is explained as follows. The non-linear regression model underlying (12) is

$$
C_{i}=C_{i}^{B S}\left(\sigma_{i}(\theta)\right)+\varepsilon_{i}
$$

The essential difference between (13) and the standard PBS model based around (2) is that while in (2) a stochastic term is applied to the IV equation, in (13) IV is treated as deterministic and the stochastic term is instead applied to the option value.

The problem with (13) is that, unless the error term $\left(\varepsilon_{i}\right)$ is identically zero, it violates the identity that defines implied volatility, namely,

$$
C_{i} \equiv C_{i}^{B S}\left(\sigma_{i}(\theta)\right)
$$

Put differently, as soon as a non-zero error term is applied to an option price, the option price becomes inconsistent with the implied volatility appearing in brackets on the RHS of (13).

This means that the implied volatility surface estimated using NLLS will not provide implied volatilities that correspond to the option prices used to estimate it. Hence the NLLS estimator of the volatility surface is inconsistent.

This may be verified by applying the Hausman (Hausman 1978) test to establish that the IV equation estimated using NLLS is systematically different from that estimated using log-linear regression on IV data. The formula for the Hausman test statistic is

$$
H=\left(\hat{\theta}_{\log I V}-\hat{\theta}_{N L L S}\right)^{\prime}\left(\hat{V}_{\log I V}-\hat{V}_{N L L S}\right)^{-1}\left(\hat{\theta}_{\log I V}-\hat{\theta}_{N L L S}\right)
$$

where $\hat{V}_{\log I V}$ and $\hat{V}_{N L L S}$ are the estimated variance matrices of the two estimators. Let $k$ be the dimension of $\theta$. If $H>\chi_{k, 0.05}^{2}$, there is evidence that $\hat{\theta}_{N L L S}$ is systematically different from $\hat{\theta}_{\log I V}$.

\section{Estimation of IV Equations Using Real Data}

In this section, we apply the various models to estimate the IV equation using data on S\&P500 call options from a single arbitrarily chosen day. The chosen day is 27 July 2000. Data on 51 call options traded on this day are available. ${ }^{4}$ The full data set is presented in Appendix A.

In Table 1, we report the estimated IV equations resulting from four different models. In Model 1 it is simply assumed that all options have the same IV, equal to the mean of IV taken over all options; this mean is 0.182 . Note that this is actually a standard PBS model with no explanatory variables in the IV equation, and is therefore a flat implied volatility surface.

One reason for selecting the chosen day as the data source is that it gives rise to an interesting implied volatility surface in the sense that most of the coefficients in the implied volatility equation are estimated as significantly different from zero. In particular, the coefficients of $K$ and $K^{2}$ (respectively significantly negative and significantly positive in all models in which they appear) represent strong evidence of the well-known "volatility smile".

Model 2 is the IV equation used in the standard PBS method, that is, an equation with IV as the dependent variable, and $K, K^{2}, \tau, \tau^{2}$, and $K \tau$ as explanatory variables. Model 3 is the same as Model 2 but with $\log (\mathrm{IV})$ as the dependent variable. Figure 1 compares predicted IV from Models 2 and 3. The two curves are broadly similar, both displaying the "smile", although perhaps interestingly, the lowest point of the latter is somewhat to the right of that of the former. Model 3 is the model in which we are most interested, since this is the model whose estimates we use as the basis of the DGP for the Monte Carlo study reported in the next section. 
Table 1. Estimation Results. Implied volatility (IV) equations from various models: (1) Linear regression with IV as dependent variable and no explanatory variables; (2) Linear regression with IV as dependent variable and standard set of explanatory variables; (3) log-linear regression with $\log (\mathrm{IV})$ as dependent variable and standard set of explanatory variables; (4) non-linear least squares estimation of $\log (\mathrm{IV})$ equation with option price as the dependent variable. Hausman test tests for systematic difference between estimates from Models 3 and 4. Data is from 51 options traded on 27 July 2000 (See Appendix A for complete data set).

\begin{tabular}{|c|c|c|c|c|}
\hline & Model 1 & Model 2 & Model 3 & Model 4 \\
\hline Variable & IV Mean Only & IV (Standard PBS) & Log(IV) (Log-Linear PBS) & NLLS (Log IV) \\
\hline K & & $\begin{array}{c}-0.00414^{* * *} \\
(-8.91)\end{array}$ & $\begin{array}{c}-0.0168^{* * *} \\
(-8.85)\end{array}$ & $\begin{array}{c}-0.00626^{* * *} \\
(-4.55)\end{array}$ \\
\hline$K^{2}$ & & $\begin{array}{l}0.00000131^{* * *} \\
(7.98)\end{array}$ & $\begin{array}{l}0.00000517^{* * *} \\
(7.72)\end{array}$ & $\begin{array}{c}0.00000159^{* *} \\
(3.27)\end{array}$ \\
\hline$\tau$ & & $\begin{array}{c}1.104^{* * * *} \\
(4.34)\end{array}$ & $\begin{array}{l}3.978^{* * *} \\
(3.83)\end{array}$ & $\begin{array}{l}-0.256 \\
(-0.38)\end{array}$ \\
\hline$\tau^{2}$ & & $\begin{array}{l}0.0262 \\
(0.49)\end{array}$ & $\begin{array}{l}-0.136 \\
(-0.62)\end{array}$ & $\begin{array}{l}-0.421^{* *} \\
(-3.48)\end{array}$ \\
\hline$K * \tau$ & & $\begin{array}{c}-0.000668^{* * *} \\
(-3.76)\end{array}$ & $\begin{array}{c}-0.00222 * * \\
(-3.06)\end{array}$ & $\begin{array}{c}0.000594 \\
(1.24)\end{array}$ \\
\hline constant & $\begin{array}{l}0.182 * * * \\
(50.33)\end{array}$ & $\begin{array}{l}3.426^{* * *} \\
(10.42)\end{array}$ & $\begin{array}{l}11.72 * * * \\
(8.73)\end{array}$ & $\begin{array}{c}4.003^{* * *} \\
(4.10)\end{array}$ \\
\hline $\begin{array}{c}\sigma \\
\mathrm{n} \\
R^{2}\end{array}$ & $\begin{array}{c}0.0259 \\
51 \\
0\end{array}$ & $\begin{array}{c}0.00692 \\
51 \\
0.936\end{array}$ & $\begin{array}{c}0.0282 \\
51 \\
0.954\end{array}$ & 51 \\
\hline Hausman $\chi^{2}(5)(p$-value $)$ & & & \multicolumn{2}{|l|}{$\begin{array}{c}516.0 \\
(0.0000)\end{array}$} \\
\hline
\end{tabular}

$t$ statistics in parentheses; ${ }^{*} p<0.05,{ }^{* *} p<0.01,{ }^{* * *} p<0.001$.

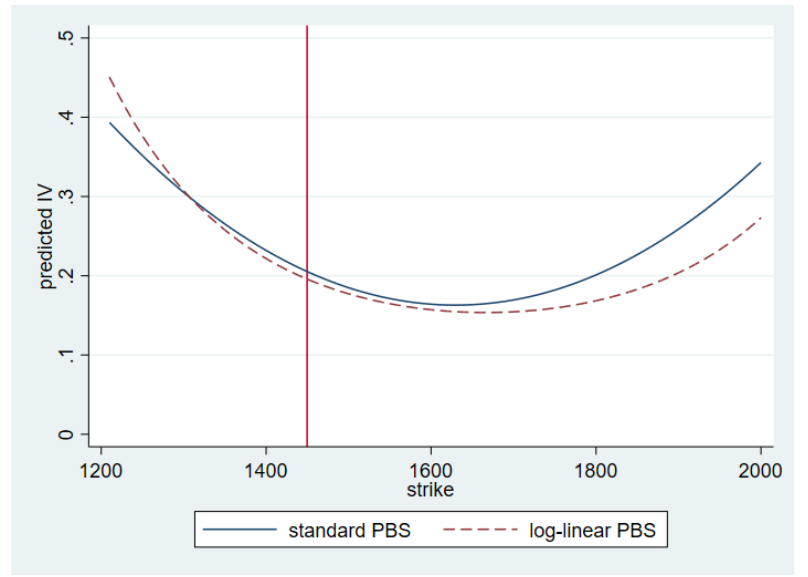

Figure 1. Predicted IV against strike price from Standard Practitioner Black-Scholes (PBS) (Model 2 in Table 1) and Log-linear PBS (Model 3 in Table 1). $\tau$ and $\tau^{2}$ fixed at sample means. Vertical line drawn at current underlying price.

It is informative to examine the residuals from Model 3. A histogram of these residuals is presented in Figure 2, with a normal distribution superimposed. The histogram suggests that the distribution of residuals is negatively skewed. With this in mind, the Monte Carlo study reported in the next section includes a DGP with a negatively skewed error. For good measure, we also include DGPs with symmetric and positively skewed errors. 


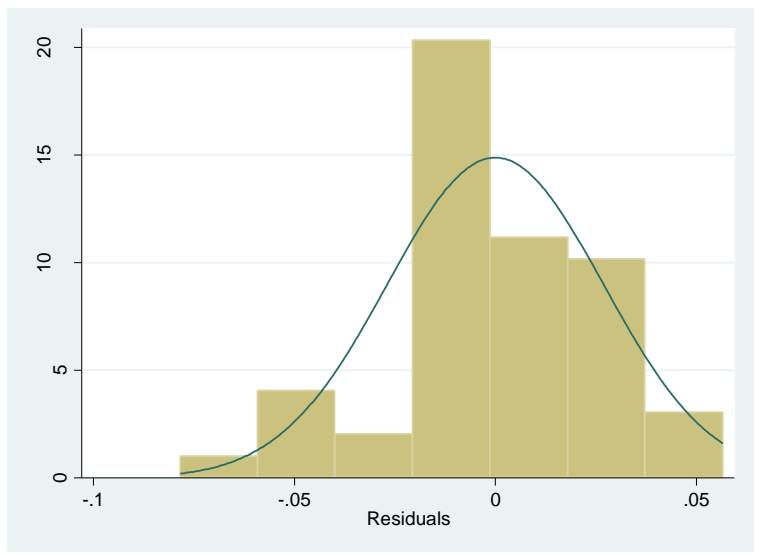

Figure 2. Histogram of residuals from log-linear model (Model 3 in Table 1). Normal density superimposed.

Model 4 applies NLLS to the problem of estimating the parameters of the log(IV) equation the using option price data. It is noted that the estimates from Model 4 appear somewhat different from those of Model 3. One reason for this difference was explained in Section 4: an incorrect equation for $\log (\mathrm{IV})$ is being estimated when NLLS is used. The Hausman test statistic has been obtained using (15) and confirms the difference: there is overwhelming evidence of a systematic difference between the two sets of estimates (Models 3 and 4), which confirms that NLLS provides an inconsistent estimate of the true implied volatility surface.

\section{A Monte Carlo Analysis of PBS with Smearing}

In this section, we set out to verify using the Monte Carlo method that the standard PBS is biased and that PBS with smearing corrects the bias.

For the design matrix (the matrix whose columns are the model's explanatory variables) we use the same set of 51 S\&P 500 (call) options as used in Section 5. We vertically concatenate this matrix with itself to give a total of 102 rows: the first block of 51 rows is used for estimation and in-sample prediction; the second block of 51 observations is used for out-of-sample prediction.

For the data generation process, we use the estimates from the third column of Table 1. That is, the data generating process for IV is

$$
\begin{gathered}
\ln \left(I V_{i}\right)=11.72-0.0168 K_{i}+0.000005 K_{i}^{2}+3.98 \tau_{i}-0.136 \tau_{i}^{2}-0.0022 K_{i} \tau_{i}+\varepsilon_{i} \\
V\left(\varepsilon_{i}\right)=0.0282^{2} \\
i=1, \ldots, 102
\end{gathered}
$$

We make three different assumptions about the distribution of the error term in (16): normal; positive skew; negative skew. For the skewed errors, we use a suitably standardised chi-squared (3) distribution. ${ }^{5}$

Having generated a sample of Implied Volatilities, the Black-Scholes formula is then applied to each of them to generate the sample of option prices.

Table 2 presents the results from the Monte Carlo analysis. For each of the three distributional assumptions, we perform 10,000 replications. We then compute the mean of the following three

5 For the positively skewed error, we simulate $\varepsilon_{i}=0.0282 \times\left(\chi^{2}(3)-3\right) / \sqrt{ } 6$. For the negatively skewed error, we apply the same formula with the sign reversed. Note that these skewed distributions have the same mean and variance as the normal error $N\left(0,0.0282^{2}\right)$ used for the symmetric case. 
quantities over the 10,000 replications, where predictions $\left(\hat{C}_{i}\right)$ for each model have been obtained both in-sample and out-of-sample:

$$
\begin{gathered}
\text { BIAS }: \frac{1}{n} \sum_{i=1}^{n}\left(\hat{C}_{i}-C_{i}\right) \\
\text { MAE }: \frac{1}{n} \sum_{i=1}^{n}\left|\hat{C}_{i}-C_{i}\right| \\
\text { MSE }: \frac{1}{n} \sum_{i=1}^{n}\left(\hat{C}_{i}-C_{i}\right)^{2}
\end{gathered}
$$

Table 2. Monte Carlo Results. Models correspond to Models 1-4 in Table 1. The data generating process (DGP) combines Equation (15) and design matrix presented in Appendix A. Distribution of error is explained in footnote 5. There are 10,000 Replications for each result set. Means over 10,000

\begin{tabular}{|c|c|c|c|c|}
\hline & (1) PBS MEAN & (2) PBS IV (Standard PBS) & (3) PBS LogIV SMEARING & (4) NLLS \\
\hline \multicolumn{5}{|c|}{ Symmetric error: } \\
\hline \multicolumn{5}{|c|}{ In sample: } \\
\hline BIAS & 0.0972 & 0.0127 & 0.0005 & -0.0031 \\
\hline MAE & 2.4102 & 0.6714 & 0.6428 & 0.6324 \\
\hline MSE & 9.5759 & 0.9086 & 0.8262 & 0.7435 \\
\hline \multicolumn{5}{|c|}{ Out of sample: } \\
\hline BIAS & 0.0937 & 0.0092 & -0.0033 & -0.0067 \\
\hline MAE & 2.4147 & 0.7640 & 0.7413 & 0.7681 \\
\hline MSE & 9.6238 & 1.2787 & 1.1951 & 1.2787 \\
\hline \multicolumn{5}{|c|}{ Negatively skewed error: } \\
\hline \multicolumn{5}{|c|}{ In sample: } \\
\hline BIAS & 0.0965 & 0.0129 & 0.0007 & -0.0028 \\
\hline MAE & 2.4081 & 0.6269 & 0.5983 & 0.5891 \\
\hline MSE & 9.5111 & 0.8620 & 0.7798 & 0.7017 \\
\hline \multicolumn{5}{|c|}{ Out of sample: } \\
\hline BIAS & 0.0979 & 0.0143 & 0.0018 & -0.0015 \\
\hline MAE & 2.4132 & 0.7096 & 0.6891 & 0.7118 \\
\hline MSE & 9.5551 & 1.2064 & 1.1290 & 1.1948 \\
\hline \multicolumn{5}{|c|}{ Positively skewed error: } \\
\hline \multicolumn{5}{|c|}{ In sample: } \\
\hline BIAS & 0.0974 & 0.0127 & 0.0005 & -0.0033 \\
\hline MAE & 2.4137 & 0.6510 & 0.6188 & 0.6113 \\
\hline MSE & 9.6282 & 0.9629 & 0.8786 & 0.7908 \\
\hline \multicolumn{5}{|c|}{ Out of sample: } \\
\hline BIAS & 0.0959 & 0.0112 & -0.0013 & -0.0049 \\
\hline MAE & 2.4176 & 0.7385 & 0.7119 & 0.7120 \\
\hline MSE & 9.6708 & 1.3516 & 1.2623 & 1.3573 \\
\hline
\end{tabular}
replications taken: bias (of predicted option prices); mean absolute error (MAE); mean squared error (MSE). Results were obtained using simulate command in STATA with random number seed 87654321.

Because the focus of the paper is correcting the bias of PBS, we will start by focusing on the bias. We first see in Table 2 that standard PBS (PBS IV) always has a considerably smaller bias than the PBS mean. This simply tells us, unsurprisingly, that a model that assumes that the volatility surface is flat (i.e., PBS mean) leads to severely biased prediction of option prices. However, we also see that the bias of PBS with smearing is-again in every case-considerably lower than the bias of standard PBS. This is exactly the result we set out to verify.

Turning to NLLS, we see that the bias of NLLS is also lower than that of standard PBS, but the bias of NLLS is larger than that of PBS with smearing in every case but one. Broadly, on the criterion of unbiasedness, PBS with smearing is the best method of the ones considered. 
Clearly we are not only interested in the bias-we are also interested in precision. Standard measures of precision are MAE and MSE defined in (17) above. In Table 2, we see that the MSE of PBS with smearing is-yet again in every case-lower than that of standard PBS. NLLS, when used to predict in-sample, tends to have the smallest MAE and MSE. However, in the perhaps more important setting of out-of-sample prediction, PBS with smearing has the smallest MAE and MSE.

Overall, we may conclude that, of the prediction methods considered, PBS with smearing is preferred because it is the closest to being unbiased, and it also has the highest precision when used to predict out-of-sample.

Finally, we can use the Monte Carlo experiment to verify that NLLS estimates the incorrect IV surface, as postulated in Section 4. In Section 5, in the context of a single sample, the Hausman test was used to establish that the NLLS estimate was systematically different from the estimate from log-linear regression. In the context of the Monte Carlo study, a more direct test is possible. We apply Wald Testing procedure ${ }^{6}$ to test the null hypothesis that the parameters estimated by NLLS equal the "true" parameters of the IV equation which appear in (16). Such a test is performed for each replication, and then we compute the proportion of the tests that result in rejection of the null hypothesis. The results are presented in Table 3.

Table 3. Further Monte Carlo Results. Proportion of replications in which null hypothesis is rejected by Wald Test. Null Hypothesis is that each parameter equals its true value under the DGP. Nominal test size 0.05 , with 10,000 replications.

\begin{tabular}{lcc}
\hline Distributional Assumption & $\mathbf{P}\left(\right.$ Reject $\mathbf{H}_{\mathbf{0}}$ ) with Log-Linear Regression & P(Reject $\left.\mathbf{H}_{\mathbf{0}}\right)$ with NLLS \\
\hline Symmetric error & 0.052 & 0.473 \\
Negatively skewed error & 0.053 & 0.396 \\
Positively skewed error & 0.054 & 0.399 \\
\hline
\end{tabular}

For good measure, we conduct a similar test (F-test) using the estimates from the log-linear regression model. The first column contains the proportion of rejections of this test, and the numbers in this column simply tells us that the F-test has actual size close to nominal size (0.05), whether or not the error is normally distributed. The second column contains the proportion of rejections of the Wald Test when NLLS is used. These numbers are clearly much higher, and tell us that in nearly half of the simulated samples, there was sufficient evidence to conclude that the NLLS estimates were significantly different from the true values. These numbers can be interpreted in terms of test power. Clearly power is limited by the low sample size of 52, and power could be increased by raising the sample size.

The numbers in the second column of Table 3 tell us that it is reasonably likely that the NLLS estimates will be significantly different from the "true" parameters of the IV equation, even with a modest sample size.

\section{Conclusions}

This paper has, for the first time, addressed the problem of bias in the standard application of the Practitioner Black-Scholes method. The bias arises from the fact that the method uses a non-linear transformation of predictions from a linear regression model. The method used to correct this bias is the smearing method introduced by Duan (1983). The smearing method is non-parametric and has been proved by Duan to yield consistent predictions under a range of distributional assumptions.

The Monte-Carlo method has been used to verify that the application of smearing to the PBS method does indeed correct the bias. The bias of the predictions from PBS with smearing is considerably

6 The Wald test can be applied following estimation of a NLLS model in STATA by using the test command immediately after the nl command. 
lower than that of standard PBS, both in-sample and out-of-sample, and we have verified that this result is robust to distributional assumptions.

The Monte Carlo approach is clearly very useful for addressing the type of question of interest here. This is partly because the "true" model determining option prices is known, enabling clear measurement of the prediction bias from the models of interest.

It must be said that NLLS applied to option prices is another approach that performs well on the criteria considered. However, in Section 4 we raised a separate problem with NLLS-incoherency. This problem arises because the manner in which the random error term is introduced causes the option prices to be inconsistent with the implied volatilities appearing in the same model. This essentially means that the implied volatility surface estimated under NLLS is incorrect. This was verified in the Monte Carlo study. However, despite this problem, NLLS results in option price predictions that are close to being unbiased and relatively precise.

This leads us to the recommendation that if the implied volatility surface is the focus of the analysis, NLLS on option prices should be avoided, and log-linear regression should be used. If the focus is instead on accurate prediction of option prices, NLLS can reliably be used. However, log-linear PBS with smearing has been shown to perform even better under most of the situations considered.

Author Contributions: Conceptualisation and Methodology: P.G.M.; Data Curation, Software and Formal Analysis: Y.Y.; Writing: P.G.M. and Y.Y.

Funding: This research received no external funding.

Conflicts of Interest: The authors declare no conflict of interest.

Data Availability Statement: The Data that support the findings of this study are openly available in Appendix A of the paper.

\section{Appendix A Data Used for Single Day Estimation in Section 5}

Table A1. Data on 51 call options traded on 27 July 2000. $C$ is price of option (mid-point between bid and ask); $S$ is current price of underlying (S\&P 500 index); $K$ is strike price; $r f$ is risk-free rate; tau is time to expiry in years; $I V$ is implied volatility. Data Source: https:/optionmetrics.com/data-products/.

\begin{tabular}{ccccccccc}
\hline $\boldsymbol{t}$ & Date & Expirydate & $\boldsymbol{C}$ & $\boldsymbol{S}$ & $\boldsymbol{K}$ & $\boldsymbol{r f}$ & tau & iv \\
\hline 1 & 27 July 2000 & 16 December 2000 & 40.5 & 1449.62 & 1550 & 0.0598 & 0.3890411 & 0.18459306 \\
2 & 27 July 2000 & 16 September 2000 & 2.875 & 1449.62 & 1600 & 0.0598 & 0.139726 & 0.16379156 \\
3 & 27 July 2000 & 19 August 2000 & 8.125 & 1449.62 & 1500 & 0.0598 & 0.0630137 & 0.16414911 \\
4 & 27 July 2000 & 16 December 2000 & 7 & 1449.62 & 1700 & 0.0598 & 0.3890411 & 0.16543757 \\
5 & 27 July 2000 & 19 August 2000 & 12.625 & 1449.62 & 1485 & 0.0598 & 0.0630137 & 0.16855563 \\
6 & 27 July 2000 & 19 August 2000 & 157.125 & 1449.62 & 1300 & 0.0598 & 0.0630137 & 0.29000379 \\
7 & 27 July 2000 & 19 August 2000 & 133 & 1449.62 & 1325 & 0.0598 & 0.0630137 & 0.26658718 \\
8 & 27 July 2000 & 19 August 2000 & 26.375 & 1449.62 & 1455 & 0.0598 & 0.0630137 & 0.18138442 \\
9 & 27 July 2000 & 16 September 2000 & 14.75 & 1449.62 & 1525 & 0.0598 & 0.139726 & 0.17296152 \\
10 & 27 July 2000 & 16 September 2000 & 47.25 & 1449.62 & 1450 & 0.0598 & 0.139726 & 0.19113849 \\
11 & 27 July 2000 & 19 August 2000 & 18.625 & 1449.62 & 1470 & 0.0598 & 0.0630137 & 0.1738262 \\
12 & 27 July 2000 & 16 September 2000 & 36.125 & 1449.62 & 1470 & 0.0598 & 0.139726 & 0.18486601 \\
13 & 27 July 2000 & 21 October 2000 & 6.25 & 1449.62 & 1625 & 0.0598 & 0.2356164 & 0.16558332 \\
14 & 27 July 2000 & 19 August 2000 & 109.125 & 1449.62 & 1350 & 0.0598 & 0.0630137 & 0.24189121 \\
15 & 27 July 2000 & 19 August 2000 & 1.625 & 1449.62 & 1545 & 0.0598 & 0.0630137 & 0.15661302 \\
16 & 27 July 2000 & 19 August 2000 & 11.125 & 1449.62 & 1490 & 0.0598 & 0.0630137 & 0.16827447 \\
17 & 27 July 2000 & 16 December 2000 & 31.75 & 1449.62 & 1575 & 0.0598 & 0.3890411 & 0.18067305 \\
18 & 27 July 2000 & 19 August 2000 & 14.5 & 1449.62 & 1480 & 0.0598 & 0.0630137 & 0.17055967 \\
19 & 27 July 2000 & 19 August 2000 & 3.6875 & 1449.62 & 1525 & 0.0598 & 0.0630137 & 0.1615991 \\
20 & 27 July 2000 & 17 March 2001 & 61.375 & 1449.62 & 1575 & 0.0598 & 0.6383561 & 0.19218853 \\
21 & 27 July 2000 & 16 December 2000 & 13.5 & 1449.62 & 1650 & 0.0598 & 0.3890411 & 0.17021512 \\
\hline
\end{tabular}


Table A1. Cont.

\begin{tabular}{|c|c|c|c|c|c|c|c|c|}
\hline$t$ & Date & Expirydate & C & $S$ & $K$ & $r f$ & tau & $i v$ \\
\hline 22 & 27 July 2000 & 19 August 2000 & 1.3125 & 1449.62 & 1550 & 0.0598 & 0.0630137 & 0.15584699 \\
\hline 23 & 27 July 2000 & 16 September 2000 & 63.625 & 1449.62 & 1425 & 0.0598 & 0.139726 & 0.20124521 \\
\hline 24 & 27 July 2000 & 16 September 2000 & 38.875 & 1449.62 & 1465 & 0.0598 & 0.139726 & 0.18695045 \\
\hline 25 & 27 July 2000 & 19 August 2000 & 16.5 & 1449.62 & 1475 & 0.0598 & 0.0630137 & 0.17231101 \\
\hline 26 & 27 July 2000 & 17 March 2001 & 5.125 & 1449.62 & 1850 & 0.0598 & 0.6383561 & 0.16320811 \\
\hline 27 & 27 July 2000 & 16 December 2000 & 62.625 & 1449.62 & 1500 & 0.0598 & 0.3890411 & 0.19390107 \\
\hline 28 & 27 July 2000 & 16 September 2000 & 23 & 1449.62 & 1500 & 0.0598 & 0.139726 & 0.17839537 \\
\hline 29 & 27 July 2000 & 19 August 2000 & 31.875 & 1449.62 & 1445 & 0.0598 & 0.0630137 & 0.18350645 \\
\hline 30 & 27 July 2000 & 16 September 2000 & 1.5 & 1449.62 & 1625 & 0.0598 & 0.139726 & 0.1619217 \\
\hline 31 & 27 July 2000 & 19 August 2000 & 35 & 1449.62 & 1440 & 0.0598 & 0.0630137 & 0.18568564 \\
\hline 32 & 27 July 2000 & 16 December 2000 & 4.75 & 1449 & 1725 & 0.0 & 0.389 & 0.16223686 \\
\hline 33 & 27 July 2000 & 16 December 2000 & 3.125 & 1449.62 & 1750 & 0.0598 & 0.3890411 & 0.15914554 \\
\hline 34 & 27 July 2000 & 16 December 2000 & 9.5 & 1449.62 & 1675 & 0.0598 & 0.3890411 & 0.16608682 \\
\hline 35 & 27 July 2000 & 16 September 2000 & 19.375 & 1449.62 & 1510 & 0.0598 & 0.139726 & 0.17603378 \\
\hline 36 & 27 July 2000 & 19 August 2000 & 2.0625 & 1449.62 & 1540 & 0.0598 & 0.0630137 & 0.15860899 \\
\hline 37 & 27 July 2000 & 16 December 2000 & 18.125 & 1449.62 & 1625 & 0.0598 & 0.3890411 & 0.17263715 \\
\hline 38 & 27 July 2000 & 19 August 2000 & 29.125 & 1449.62 & 1450 & 0.0598 & 0.0630137 & 0.18295443 \\
\hline 39 & 27 July 2000 & 16 September 2000 & 81.125 & 1449.62 & 1400 & 0.0598 & 0.139726 & 0.20730415 \\
\hline 40 & 27 July 2000 & 21 October 2000 & 20.625 & 1449.62 & 1550 & 0.0598 & 0.2356164 & 0.17530438 \\
\hline 41 & 27 July 2000 & 16 September 2000 & 33.875 & 1449.62 & 1475 & 0.0598 & 0.139726 & 0.1846462 \\
\hline 42 & 27 July 2000 & 16 September 2000 & 100.625 & 1449.62 & 1375 & 0.0598 & 0.139726 & 0.21527798 \\
\hline 43 & 27 July 2000 & 16 December 2000 & 24.25 & 1449.62 & 1600 & 0.0598 & 0.3890411 & 0.1764836 \\
\hline 44 & 27 July 2000 & 19 August 2000 & 64.75 & 1449.62 & 1400 & 0.0598 & 0.0630137 & 0.2074976 \\
\hline 45 & 27 July 2000 & 19 August 2000 & 3 & 1449.62 & 1530 & 0.0598 & 0.0630137 & 0.15969375 \\
\hline 46 & 27 July 2000 & 21 October 2000 & 65.625 & 1449.62 & 1450 & 0.0598 & 0.2356164 & 0.19772039 \\
\hline 47 & 27 July 2000 & 16 September 2000 & 5.125 & 1449.62 & 1575 & 0.0598 & 0.139726 & 0.16519108 \\
\hline 48 & 27 July 2000 & 16 September 2000 & 8.875 & 1449.62 & 1550 & 0.0598 & 0.139726 & 0.16814606 \\
\hline 49 & 27 July 2000 & 19 August 2000 & 45.25 & 1449.62 & 1425 & 0.0598 & 0.0630137 & 0.19296223 \\
\hline 50 & 27 July 2000 & 17 March 2001 & 35.625 & 1449.62 & 1650 & 0.0598 & 0.6383561 & 0.18229888 \\
\hline 51 & 27 July 2000 & 16 September 2000 & 41.5 & 1449.62 & 1460 & 0.0598 & 0.139726 & 0.18801008 \\
\hline
\end{tabular}

\section{Appendix B}

The STATA code required to apply smearing to PBS is as follows. The first three commands perform the (log-linear) IV regression and obtain the fitted values (log_ivhat), and the residuals (uhat). The main part of the code is a loop over the sample (using the forvalues command). The predicted option price is stored in the variable pbs_smearing.

regress log_iv K K2 tau tau2 Ktau

predict log_ivhat, $\mathrm{xb}$

predict uhat, resid

gen $d 1=$.

gen $\mathrm{d} 2=$.

gen pbs_smearing=.

quietly\{

local N=_N

forvalues $j=1(1)$ ' $N$ ' \{

scalar log_ivhat_temp=log_ivhat in ' $j$,

scalar S_temp=S in ' $j$,

scalar K_temp=K in ' $\mathrm{j}$ ' 


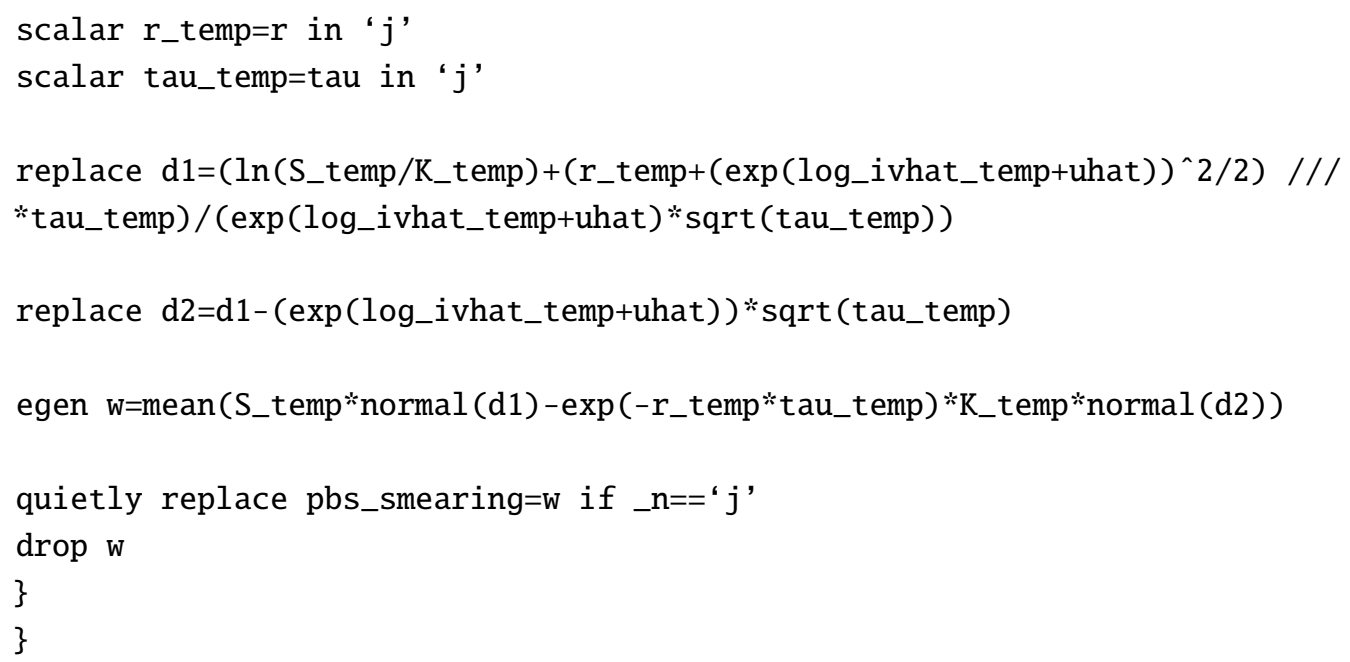

\section{References}

Andreou, Panayiotis C., Chris Charalambous, and Spiros H. Martzoukos. 2014. Assessing the performance of symmetric and asymmetric implied volatility functions. Review of Quantitative Finance and Accounting 42: 373-97. [CrossRef]

Bakshi, Gurdip, Charles Cao, and Zhiwu Chen. 1997. Empirical performance of alternative option pricing models. The Journal of Finance 52: 2003-49. [CrossRef]

Christoffersen, Peter, and Kris Jacobs. 2004. The importance of the loss function in option valuation. Journal of Financial Economics 72: 291-318. [CrossRef]

Duan, Naihua. 1983. Smearing estimate: A nonparametric retransformation method. Journal of the American Statistical Association 78: 605-10. [CrossRef]

Duan, J., Genevieve Gauthier, J. Simonato, and Caroline Sasseville. 2006. Approximating the GJR-GARCH and EGARCH option pricing models analytically. Journal of Computational Finance 9: 41. [CrossRef]

Dumas, Bernard, Jeff Fleming, and Robert E. Whaley. 1998. Implied volatility functions: Empirical tests. The Journal of Finance 53: 2059-106. [CrossRef]

Hausman, Jerry A. 1978. Specification tests in econometrics. Econometrica 46: 1251-71. [CrossRef]

Heston, Steven L., and Saikat Nandi. 2000. A closed form GARCH option pricing model. The Review of Financial Studies 13: 585-625. [CrossRef]

Hull, John. 2011. Fundamentals of Futures and Options Markets, 7th ed. London: Pearson.

Yin, Y. 2018. Flexible Option Valuation Methods. Unpublished M.Phil. thesis, University of East Anglia, Norwich, UK. 